\title{
Numerical Calculation of Viscous-Elastic Fluid Flooding Residual Oil Film in the Complex Pore
}

\author{
Lili Liu ${ }^{1}$, Chao Yu ${ }^{1}$, Lihui Wang ${ }^{2}$, Lu Liu ${ }^{1}$ \\ ${ }^{1}$ Department of Petroleum Engineering, Northeast Petroleum University, Daqing, China \\ ${ }^{2}$ Daqing Petroleum Equipment Group, Daqing, China \\ Email: dqliull2009@163.com
}

Received May 14, 2013; revised June 13, 2013; accepted June 27, 2013

Copyright (c) 2013 Lili Liu et al. This is an open access article distributed under the Creative Commons Attribution License, which permits unrestricted use, distribution, and reproduction in any medium, provided the original work is properly cited.

\begin{abstract}
In order to analyze the stress and deformation of different permeability of residual oil film in the complex pore, which are affected by the viscous-elasticity of the fluid, the hydrodynamic displacement mechanism is explored from the standpoint of hydrodynamics, that is, the residual oil film displaced by alternating injection of different concentrations of the polymer solution, viscous-elastic fluid flow equation is established in the complex pore by choosing continuity equation, motion equation and the upper convected Maxwell constitutive equation. The flow field is computed by using the method of numerical analysis. Not only the stress and deformation of residual oil film on the different permeability of micro pores, but also the analysis of the flooding mechanism of alternating injection of different concentrations of the polymer solution is got. The results show that the larger the viscous-elasticity of polymer solution is, the bigger the normal deviatoric stress acting on the residual oil film is; the distribution of normal deviatoric stress has the abrupt change. The stronger the viscous-elasticity of the polymer solution is, the bigger the horizontal stress difference acting on the residual oil film is and the more obvious the deformation is; the high-concentration polymer solution is suitable for high-permeability micro pores. Low-concentration polymer solution is suitable for medium and low-permeability micro pores. Alternating injection of polymer solution can improve Volumetric Sweep Efficiency and increase the deformation of residual oil film, which is conducive to enhancing oil recovery.
\end{abstract}

Keywords: Complex Pore; Residual Oil Film; Deformation; Viscous-Elasticity; Permeability

\section{Introduction}

After water flooding, polymer flooding has a favourable effect on flooding effects, which largely enhance the oil recovery [1]. Because of stratigraphic shearing and dilution of the formation water, simple injection of highconcentration polymer makes the plugging effect decrease with time. Because high-permeability layer is very easy to break, simple injection of low-concentration polymer solution makes low concentration of polymer crossflow in high-permeability pore, hardly get into medium and low-permeability layer. In order to solve the drawbacks of general injection method, alternating injection is taken to control mobility well. Each permeable layer takes what he needs. Swept volume is also expanded. Zhang Jihong [2] and others got results from experiment that alternating injection of gel and surfactant flooding after polymer flooding was possible to further enhance oil recovery. Zhang Zhilong [3] and others explored flooding situation of alternating injection of foam and AS after polymer flooding. In this paper, the force and deformation of residual oil film acted by polymer solution are analyzed theoretically and the result that alternating injection of different concentrations of the polymer solution can also enhance oil recovery.

\section{Calculation Model}

In order to analyze the force and deformation of the residual oil film in different permeability micro pores, Two-dimensional parallel channel model is shown in Figure 1. Low, medium and the high micro pores width, respectively, are taken as $10 \mu \mathrm{m}, 20 \mu \mathrm{m}, 30 \mu \mathrm{m}$, taking micro pores width proportion $H_{1}: H_{2}: H_{3}$ as 1:2:3. Figure $\mathbf{2}$ is the corresponding calculation model.

In Figure 2, both model building and grid designing are taken account into inlet effect and boundary effect.

\section{Basic Equation}

Polymer solution with complicated rheological proper 


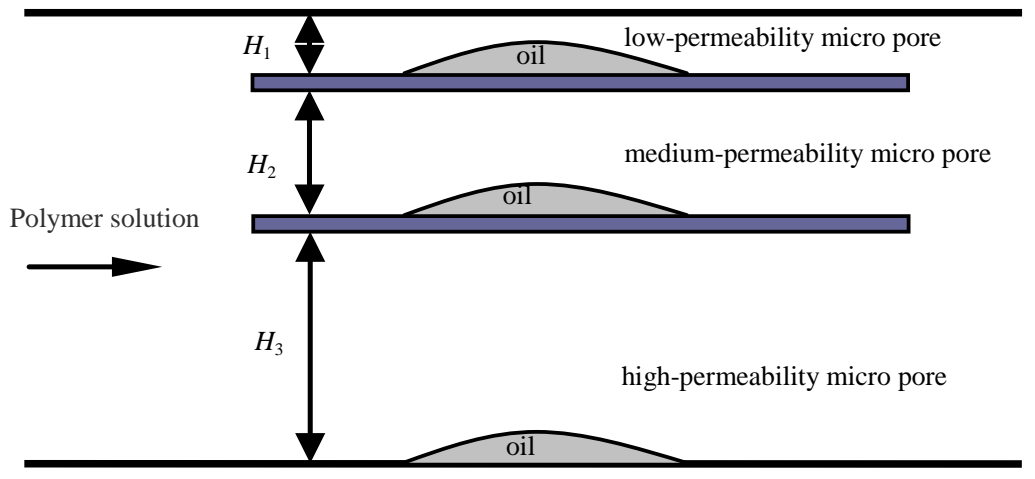

Figure 1. Parallel channel model.

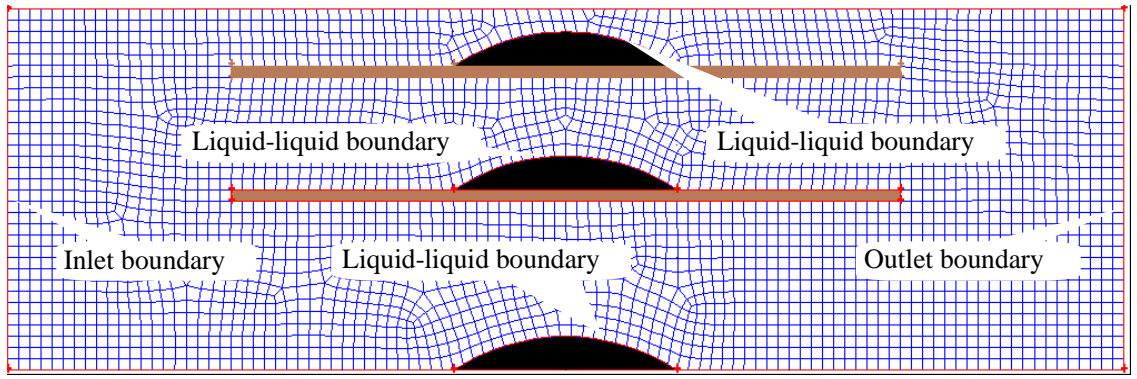

Figure 2. Computational grid and boundary condition

belongs to viscous-elastic fluid. Based on experiment research, the upper convected Maxwell constitutive equation is suitable to describe the viscous-elastic polymer solution. Continuity equation, motion equation and constitutive equation [4] are used as follows:

Continuity equation:

$$
\nabla \cdot \boldsymbol{u}=0
$$

where: $\boldsymbol{u}$ is velocity vector.

Momentum equation:

$$
\mathrm{d} \boldsymbol{u} / \mathrm{d} t=\boldsymbol{F}+\operatorname{div} \boldsymbol{P} / \rho
$$

where: $\boldsymbol{F}$ is mass force; $\rho$ is density; $\boldsymbol{P}$ is stress tensor.

$$
\boldsymbol{P}=-p \boldsymbol{I}+\boldsymbol{T}
$$

where: $\boldsymbol{I}$ is unit tensor; $-p$ is static pressure; $\boldsymbol{T}$ is deviatoric stress tensor.

Constitutive equation:

$$
\boldsymbol{T}+\lambda \stackrel{\nabla}{\boldsymbol{T}}=\eta \boldsymbol{A}_{1}
$$

where: $\lambda$ is relaxation time, which is the characterization of fluid elasticity; $\eta$ is shearing viscosity; $\boldsymbol{A}_{\mathbf{1}}$ is one order Rivlin-Ericksen tensor.

\section{Calculation Result}

Weissenberger number is a dimensionless quantity which is used to describe elasticity of fluid. The bigger its value is, the stronger the elasticity of the fluid is. Calculation formula of Weissenberger number is:

$$
W e=\lambda v / l
$$

where: We is Weissenberger number; $v$ is characteristic velocity; $l$ is characteristic length.

In order to analyze the force acting on the oil film from fluid of different viscous-elastic flowing through different permeability micro pores, the stress field is calculated when viscous-elastic fluid flows residual oil film under the different conditions that $W e$ is $0.1,0.2,0.3$ respectively. In addition, normal deviatoric stress $\tau_{\mathrm{nn}}$ and the difference of horizontal stress $p_{\text {nh }}$ on the residual oil film surface are calculated.

\subsection{The Change of the Normal Deviatoric Stress [5] Acting on the Residual Oil Film in Different Permeability Cores}

As shown in Figure 3, in the different permeability micro pores, the higher of We of viscous-elastic fluid is, the larger normal deviatoric stress is; with the increase of We, the higher compression on upstream of residual oil film is, the lower tension on downstream of residual oil film is. When $W e=0.3$, in the downstream of oil film, only a small area of close to oil film center is in tension, and others are all in larger compression. The result of two ends compressed and center tensioned makes the small area of oil center extrude from oil film. Under the continual action of polymer solution, this small oil film will be pulled, it won't rupture until it achieves stress limit, finally, separate from ontology. Compared with the low- 


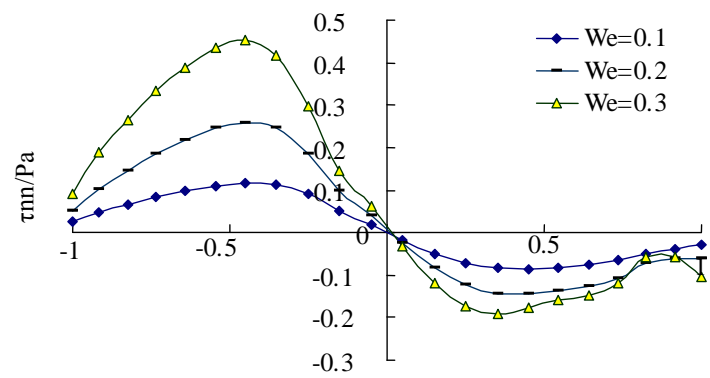

Horizontal coordinate $10 \mu \mathrm{m}$

(a)

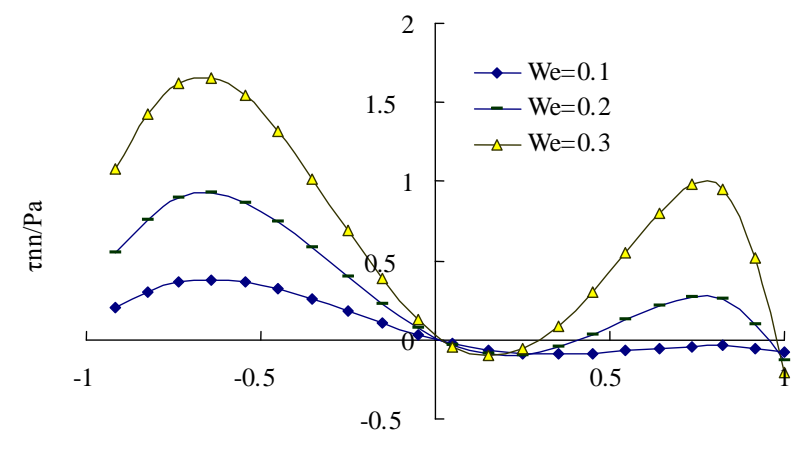

Horizontal coordinate $/ 10 \mu \mathrm{m}$

(b)

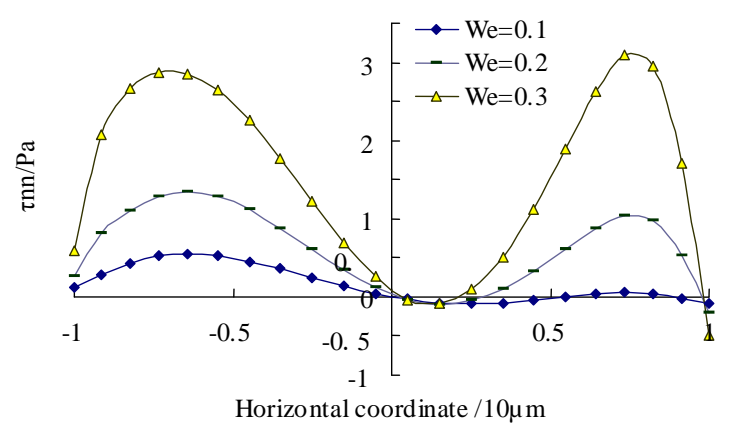

(c)

Figure 3. Normal deviatoric stress acting on residual oil film in the different permeability micro pores. (a) lowpermeability micro pore; (b) medium-permeability micro pore; (c) high-permeability micro pore. Note: compression is positive, tension is negative.

permeability micro pore, the normal deviatoric stress of high-permeability micro pore is 6 times than that of it with the same oil film.

When the polymer solution of the $W e=0.3$ is injected, the normal deviatoric stress acting on residual oil film differ greatly from low and medium-permeability micro pores from high-permeability micro pores. High viscouselasticity of the high-concentration of polymer significantly increase the force and deformation of the residual oil film, but high concentration makes polymer molecules overlap and molecular clew size large, which makes them difficultly enter the medium and low-permeability layer, especially in low-permeability layer.
Therefore, the high concentration of high molecular weight polymers mainly enriched in the high-permeability layer, generating large normal deviatoric stress to the residual oil film in high-permeability layer. This shows that the force and deformation of residual oil film in the micro pore are affected by viscous-elasticity of driving agent. High-concentration of polymer is available for high-permeability micro pore.

When the polymer solution of the $W e=0.3$ is injected, the normal deviatoric stress acting on residual oil film is less the same between low and medium-permeability micro pore and high-permeability micro pore. This is due to the low-concentration polymer solution can be relatively easy to enter the low-permeability layer. Compared to the high-concentration polymer solution, the total amount of the low-concentration polymer solution entered is large. Due to the increase in the amount of the polymer entered low and medium-permeability micro pore, the degree of deformation increases. So the low-concentration polymer is suitable for low-permeability micro pore.

\subsection{Changes of Horizontal Stress Difference Acting on the Residual Oil Film in Different Permeability Cores}

Both the microscopic visualization flooding result [6] and the theoretical result [7] showed that viscous-elastic polymer solution generated a greater level of thrust on residual oil film. The main reason for the deformation of oil film is stress difference which is produced by asymmetry of normal deviatoric stress of the film surface. Therefore, it is necessary to further analyze horizontal thrust of flooding by different driving fluids, as it is the size of the horizontal stress difference. The greater the value is, the more obvious the film deformation is. As seen from Figure 4, the horizontal stress difference of the viscous-elastic fluid increases with the increasing of We. Horizontal stress difference which high concentration of the polymer solution acting on the high-permeability oil film is significantly higher than that of low-permeability of oil film, the highconcentration polymer is suitable for high-permeability micro pore. Horizontal stress difference which low-concentration polymer acted on oil film in the medium-permeability micro pore has little difference. So the lowconcentration polymer is suitable for low-permeability micro pore.

\subsection{Deformation of the Residual Oil Film in the Different Permeability Cores [8]}

As can be seen from Figure 5, high concentration polymer solution is injected; the deformation of high-permeability of the residual oil film is more evident than that of the low and medium-permeability. The deformation of residual oil film of high, medium and low-permeability 


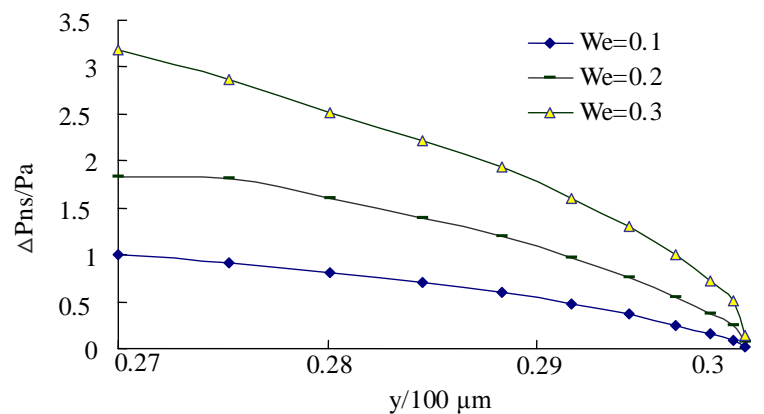

(a)

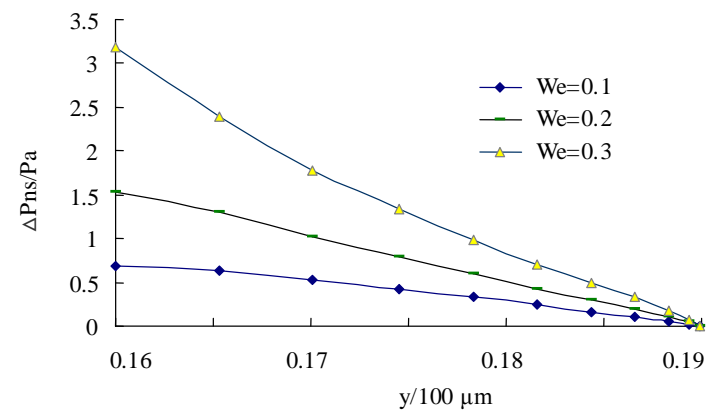

(b)

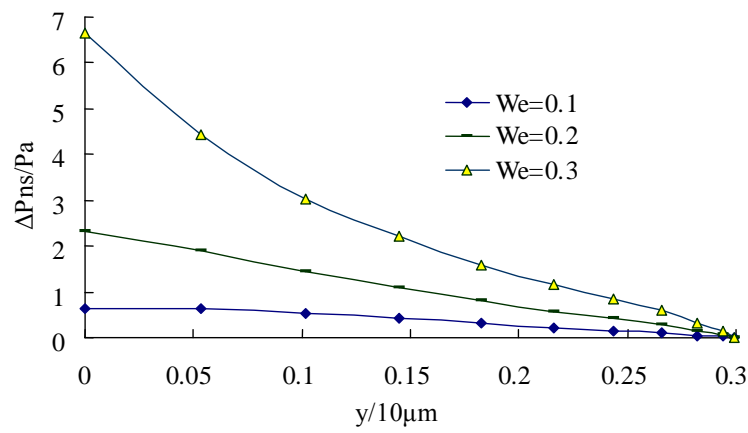

(c)

Figure 4. Horizontal stress difference acting on the residual oil film in different permeability cores. (a) low-permeability micro pore; (b) medium-permeability micro pore; (c) highpermeability micro pore.

have little difference when low concentration of polymer solution is injected.

Through the analysis of the force and deformation of residual oil film, we find that alternating injection different viscous-elastic driving fluid can enhance oil recovery. General injection is taken, force and deformation of the residual oil is very small in low and medium-permeability micro pore, displacing effect is not good, the degree of use is low; force and deformation of the residual oil is large in high-permeability micro pore. Therefore, alternating injection takes advantage of principle that different driving agent flooding will make different force and deformation of the residual oil, which can improve excessive absorption of fluid in high-permeability micro pore and insufficient absorption of fluid in low and me-

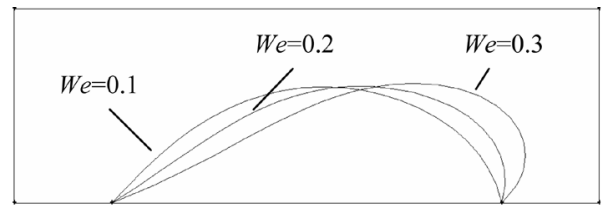

(a)

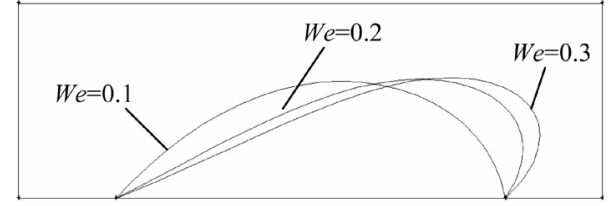

(b)

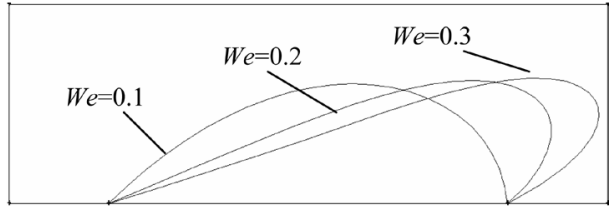

(c)

Figure 5. Deformation of the residual oil film in the different permeability micro pore. (a) low-permeability micro pore; (b) medium-permeability micro pore; (c) high-permeability micro pore.

dium-permeability micro pore, make more low-concentration polymer into low and medium-permeability micro pore, Each permeability layer takes what he needs; fluidity is controlled well, the residual oil of force and deformation is more, and the swept volume is expanded, at the same time, it will play a better role in flooding.

\section{Conclusions}

- The normal deviatoric stress of viscous-elastic polymer solution acting on oil film increases with the increasing of We. The distribution of normal deviatoric stress occurs corresponding to mutations, which are more conducive to the deformation of the oil film.

- The horizontal stress difference of driving fluid acting on oil film increases with the elasticity of driving fluid increased.

- For high-permeability micro pore, high-concentration polymer solution is injected which can acquire the largest normal deviatoric stress and the most obvious deformation. For low and medium-permeability micro pore, the normal deviatoric stress of injecting lowconcentration polymer solution is more or less the same with that in the high-permeability micro pore.

- Alternating injection different viscous-elasticity polymer solution can enhance oil recovery, which is consistent with the results of laboratory experiments.

\section{REFERENCES}

[1] X. G, Yue, Y. F. Wang, K. L. Wang, et al., "Enhance Oil Recovery Foundation,” Publishing House of Oil Industry, 
Beijing, 2002.

[2] J. H. Zhang, X. Dong, Y. Z. Ye, et al., "Flooding Effects of Alternating Injection of Gel and Surfactant after Polymer Flooding," Journal of Daqing Petroleum Institute Contents, Vol. 34, No. 2, 2010, pp. 85-88.

[3] Z. L. Zhang and M. S. Wang, "Flooding Physical Simulation of Alternating Injection of Foam with the AS System after polymer Flooding," Oilfield Chemistry, Vol. 23, No. 2, 2006, pp. 166-172.

[4] S. F. Ha, "Constitutive Equation and Computational Analytical Theory of Non-Newtonian Fluids,” Science Press, Beijing, 2002.

[5] L. L. Liu, L. H. Wang, Y. Zhang, et al., "Mechanical
Analysis of Stress Acted on Residual Oil Film by Polymer Solution,” Xinjiang Petroleum Geology, Vol. 31, No. 5, 2010, pp. 533-535.

[6] D. M. Wang, J. C. Cheng, Q. Y. Yan, et al., "ViscousElastic Polymer Can Increase Micro Scale Displacement in Cores," SPE 63227, 2000, pp. 2-8.

[7] H. J. Yin, D. M. Wang, H. Y. Zhong, et al., "Study on Flow Behaviors of Viscoelastic Polymer Solution in Micro Pore with Dead End,” SPE 101950, 2001, pp. 4-15.

[8] X. H. Liu, “A Biomechanical Model for Simulating the Deformation of a Leukocyte Adhered to the Surface of a Blood Vessel under Steady Shear Flow," Journal of Biomedical Engineering, Vol. 20, No. 1, 2003, pp. 30-34. 